\title{
The course of the superficial peroneal nerve in relation to the ankle position: anatomical study with ankle arthroscopic implications
}

\author{
Peter A. J. de Leeuw $\cdot$ Pau Golanó • \\ Inger N. Sierevelt $\cdot$ C. Niek van Dijk
}

Received: 12 December 2009/Accepted: 16 February 2010/Published online: 12 March 2010

(C) The Author(s) 2010. This article is published with open access at Springerlink.com

\begin{abstract}
Despite the fact that the superficial peroneal nerve is the only nerve in the human body that can be made visible; iatrogenic damage to this nerve is the most frequently reported complication in anterior ankle arthroscopy. One of the methods to visualize the nerve is combined ankle plantar flexion and inversion. In the majority of cases, the superficial peroneal nerve can be made visible. The portals for anterior ankle arthroscopy are however created with the ankle in the neutral or slightly dorsiflexed position and not in combined plantar flexion and inversion. The purpose of this study was to undertake an anatomical study to the course of the superficial peroneal nerve in different positions of the foot and ankle. We hypothesize that the anatomical localization of the superficial peroneal nerve changes with different foot and ankle positions. In ten fresh frozen ankle specimens, a window, only affecting the skin, was made at the level of the
\end{abstract}

\section{P. A. J. de Leeuw $(\square)$}

Academic Medical Center, Department of Orthopaedic Surgery,

University of Amsterdam, PO Box 22700, 1100 DE Amsterdam,

The Netherlands

e-mail: p.a.deleeuw@amc.uva.nl; p.a.deleeuw@amc.nl

\section{P. Golanó}

Laborarory of Arthroscopic and Surgical Anatomy,

Department of Pathology and Experimental Therapeutics

(Human Anatomy Unit), University of Barcelona,

c/Feixa Llarga s/n (Campus Bellvitge), L'Hospitalet de

Llobregat, Barcelona 08097, Spain

e-mail:pgolano@ub.edu

\section{N. Sierevelt · C. N. van Dijk}

Department of Orthopaedic Surgery, Academic Medical Center, PO Box 22700, 1100 DE Amsterdam, The Netherlands

e-mail: i.n.sierevelt@amc.nl

C. N. van Dijk

e-mail: c.n.vandijk@amc.uva.nl anterolateral portal for anterior ankle arthroscopy in order to directly visualize the superficial peroneal nerve, or if divided, its terminal branches. Nerve movement was assessed from combined $10^{\circ}$ plantar flexion and inversion to $5^{\circ}$ dorsiflexion, standardized by the Telos stress device. Also for the 4th toe flexion, flexion of all the toes and for skin tensioning possible nerve movement was determined. The mean superficial peroneal nerve movement was $2.4 \mathrm{~mm}$ to the lateral side when the ankle was moved from $10^{\circ}$ plantar flexion and inversion to the neutral ankle position and $3.6 \mathrm{~mm}$ to the lateral side from $10^{\circ}$ plantar flexion and inversion to $5^{\circ}$ dorsiflexion. Both displacements were significant $(P<0.01)$. The nerve consistently moves lateral when the ankle is manoeuvred from combined plantar flexion and inversion to the neutral or dorsiflexed position. If visible, it is therefore advised to create the anterolateral portal medial from the preoperative marking, in order to prevent iatrogenic damage to the superficial peroneal nerve.

Keywords Superficial peroneal nerve . Ankle arthroscopy $\cdot$ Complications

\section{Introduction}

Over the last three decades, the field of ankle arthroscopy has progressed significantly. Nowadays, anterior ankle arthroscopy is routinely used for the treatment of the anterior ankle impingement syndrome and talar osteochondral defects [9, 27], although the technique can be used for a wide variety of ankle pathology.

The anterolateral portal in anterior ankle arthroscopy is created lateral to the peroneus tertius tendon or, if not present, lateral to the extensor digitorum longus tendon. 
The superficial peroneal nerve or its terminal branches, mainly the intermediate dorsal cutaneous nerve, is at risk with regard to this routine anterolateral portal $[4,9,10,18$, 19, 23] (Fig. 1).

The superficial peroneal nerve provides motor innervation to the peroneus longus and brevis muscles and passes along the lateral intermuscular septum, subsequently perforating the crural fascia to function as a sensory nerve to the vast majority of the dorsal foot (Fig. 2). After fascia perforation, this nerve is usually divided into its terminal branches; the medial dorsal cutaneous-and intermediate dorsal cutaneous nerve $[12,14]$. The level at which the superficial peroneal nerve divides into its terminal branches relatively to the level of the ankle joint was subject to numerous anatomical studies and different classification types [1, 5, 14, 19, 24]. The superficial peroneal nerve, and if divided, its terminal branches are the only nerves in the human body that can be made visible [21] (Fig. 3). Preoperative identification of this nerve should assist in preventing iatrogenic damage during foot and ankle surgery, rapid localization prior to local anaesthetic blocks, external fixator applications as well as surgical decompressions [21].

Arthroscopic surgery, as compared to open surgical approaches, offers the advantage of direct and magnified visualization of the anatomical structures, less postoperative morbidity, faster and functional rehabilitation, earlier sport resumption, and the possibility of outpatient treatment $[8,10,20]$. Despite these obvious advantages, complications do occur. The overall complication rate varies in between 8 and $17 \%$ [2, 4, 6, 8-11, 13, 15, 17, 18, 20], of which iatrogenic superficial peroneal nerve damage, related to anterolateral portal placement, is reported most frequently [4, 10, 18]. Ferkel et al. [9] reported an overall complication rate of $9 \%$, of which iatrogenic superficial peroneal nerve damage accounts for $27 \%$.

Numerous suggestions have been made to diminish these rates. Good anatomical knowledge, the use of vertical incisions only affecting the skin parallel to the tendons and neurovascular structures with subsequent blunt dissection up to the level of the joint have been postulated $[9,12]$. Furthermore, minimal endurance of distraction and tourniquet inflation within a protocol ensuring routine portal placement and usage of proper arthroscopic instrumentation are other considerations $[9,10,16,18,22,24]$. Preoperatively, the superficial peroneal nerve, or its terminal branches, can be made visible with ankle plantar flexion and inversion, while preoperatively it can be visualized with cutaneous transillumination [9, 21, 24, 25]. If visible, it is recommended to mark the course of the nerve on the

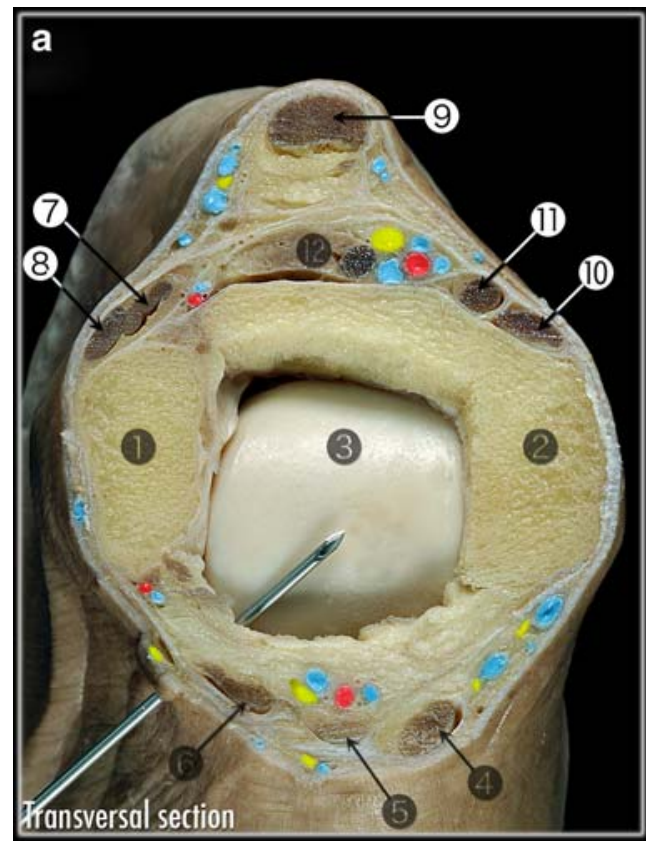

Fig. 1 Transverse section at the level of the ankle joint. a. Anatomical relations of the anterolateral portal. b. Anatomical relations of the anteromedial portal 1 Lateral malleolus, 2 Medial malleolus, 3 Talus, 4 Tibialis anterior tendon, 5 Extensor hallucis longus tendon, 6 Extensor digitorum longus and peroneus tertius tendons, 7 Peroneus brevis tendon, 8 Peroneus longus tendon, 9 Achilles tendon, 10 Tibialis posterior tendon, 11 Flexor digitorum longus, 12 Flexor hallucis tendon (musculotendinous), 13 Deep peroneal nerve and

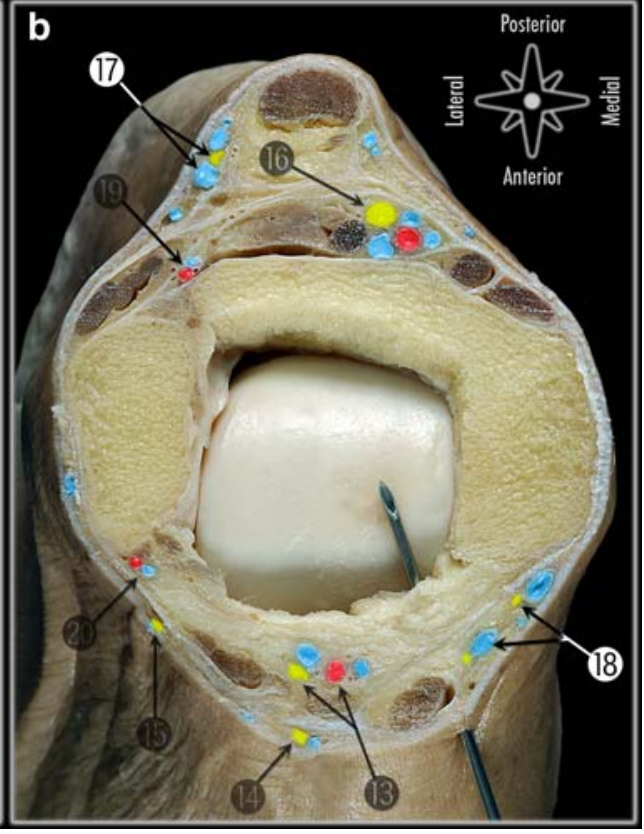

anterior tibial artery and veins, 14 Medial dorsal cutaneous nerve (medial terminal branch of superficial peroneal nerve), 15 Intermediate dorsal cutaneous nerve (lateral terminal branch of superficial peroneal nerve), 16 Posterior tibial nerve and posterior tibial artery and veins, 17 Sural nerve and small saphenous vein, 18 Saphenous nerve and great saphenous vein, 19 Posterior peroneal artery and 20 Anterior peroneal artery 


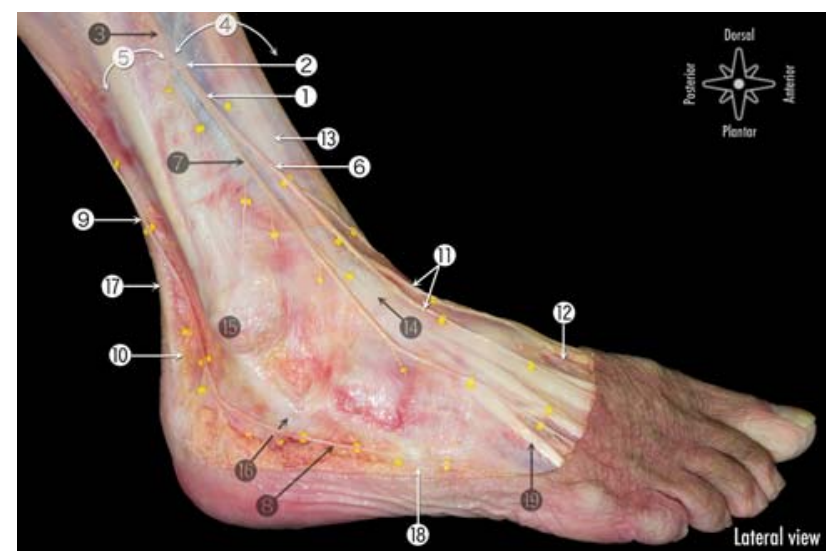

Fig. 2 Anatomical dissection of the cutaneous nerves of the foot and ankle. 1 Superficial peroneal nerve, 2 Fascial piercing of the superficial peroneal nerve, 3 Superficial peroneal nerve before piercing the crural fascia, 4 Anterior compartment of the leg, 5 Lateral compartment of the leg, 6 Medial dorsal cutaneous nerve (medial terminal branch of superficial peroneal nerve), 7 Intermediate dorsal cutaneous nerve (lateral terminal branch of superficial peroneal nerve), 8 Lateral dorsal cutaneous nerve (terminal branch of sural nerve), 9 Sural nerve (the saphenous vein was removed), 10 Medial calcaneal nerve (Branco or sural nerve), 11 Common nerves digital of medial dorsal cutaneous nerve (medial terminal branch of superficial peroneal nerve), 12 Cutaneous branch (medial terminal branch) of deep peroneal nerve, 13 Superior extensor retinaculum, 14 Inferior extensor retinaculum, 15 Tip of lateral malleolus, 16 Inferior peroneal retinaculum, 17 Achilles tendon and 18 Tuberosity of the $\mathrm{V}$ metatarsal bone

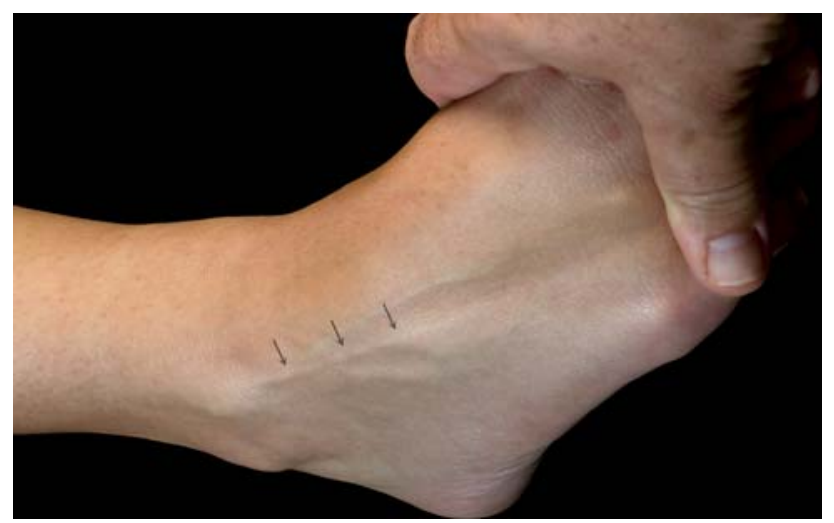

Fig. 3 Using the combined ankle plantar flexion and inversion position, the superficial peroneal nerve (the intermediate dorsal cutaneous nerve or lateral terminal branch of superficial peroneal nerve) could be visualized in 3 out of the 10 examined ankles (30\%)

skin preoperatively [9]. The effect of each intervention in reducing complication rates is unknown.

It is noteworthy that despite improved arthroscopic techniques and the fact that the superficial peroneal nerve can be visualized transcutaneously, damage to this nerve remains the most frequently reported complication in anterior ankle arthroscopy $[4,10,18]$. Therefore, the purpose of this study was to perform an anatomical study to determine the course of the superficial peroneal nerve in different positions of the foot and ankle to possibly explain and clarify this rather contradiction, and eventually to diminish future complication rates. We hypothesize that the nerve localization changes with different foot and ankle positions.

\section{Materials and methods}

Ten lower legs (five left and five right), amputated approximately $15 \mathrm{~cm}$ below the knee, from seven men and three women fresh frozen specimens were carefully examined in the Department of Pathology and Experimental Therapeutic Unit of Human Anatomy and Embryology in Barcelona, Spain. The specimens were from the Caucasian origin with a median age of 77 (range 65-90 years). Specimens were excluded in case of deformities or scars following ankle surgery and/or limited range of motion. The superficial peroneal nerve with its surrounding soft tissue was fixed with a mosquito clamp at the amputation level.

An experienced anatomist (PG) determined the course of the superficial peroneal nerve, or if divided its terminal branches, by creating a window $(20 \mathrm{~mm}$ wide and $15 \mathrm{~mm}$ long), only affecting the skin without manipulating the nerve. The localization of the window was at the level of the anterior joint line, lateral to the peroneus tertius tendons or if not present, lateral to the extensor digitorum longus tendon. Now, the superficial peroneal nerve or its terminal branches could directly be visualized. The ankle was subsequently moved from $10^{\circ}$ plantar flexion and inversion to the neutral position $\left(90^{\circ}\right)$ and then to $5^{\circ}$ dorsiflexion to determine whether the course of the nerve changed.

The reference point was regarded being the combined plantar flexion and inversion position. In this position, the medial edge of the nerve was marked with a pin ( $\varnothing 0.5 \mathrm{~mm}$ ). Possible nerve movements, relatively to the reference point, were recorded with a digital caliper (Australian measuring instruments $0-200 \mathrm{~mm}$ ). Manipulating the ankle in the different ankle positions was standardized by means of the Telos stress device (Telos equipment, Weiterstadt, Germany) (Fig. 4).

Subsequently, the ankle was positioned in the neutral position $\left(90^{\circ}\right)$ to assess whether the course of the superficial peroneal nerve, or its terminal branches, changed with manual tension on the skin covering the lateral aspect of the ankle, plantar flexion of the 4th toe and plantar flexion of all the toes.

\section{Statistical analysis}

Each of the measurements was performed by three independent observers, and inter-observer reliability was 


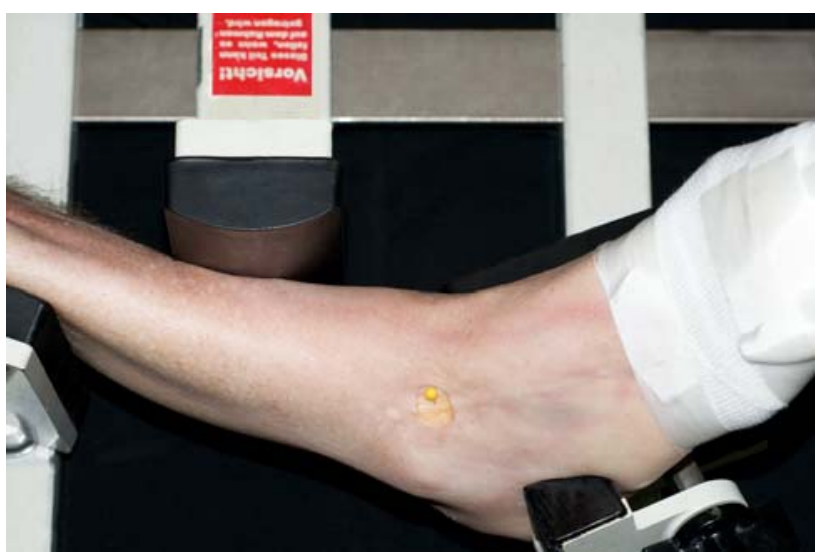

Fig. 4 Photograph showing the used method in the study. The course of the superficial peroneal nerve, or if divided its terminal branches, was determined by creating a window $(20 \mathrm{~mm}$ wide and $15 \mathrm{~mm}$ long), only affecting the skin without manipulating the nerve. Manipulating the ankle in the different ankle positions was standardized by means of the Telos stress device (Telos equipment, Weiterstadt, Germany). The reference point was regarded being the combined plantar flexion and inversion position. In this position, the medial edge of the nerve was marked with a pin (yellow in the photography)

assessed through an intraclass correlation coefficient (ICC). The reliability was considered good if the coefficient exceeded 0.8. An one sample $t$ test was performed to determine whether the nerve movement was significantly different from $0(P<0.05)$.

\section{Results}

The superficial nerve or its terminal branches consistently moved to lateral while the ankle was moved from $10^{\circ}$ plantar flexion and inversion to the neutral and then to the $5^{\circ}$ dorsiflexion position. From $10^{\circ}$ plantar flexion and inversion to the neutral position, the average nerve movement was $2.4 \mathrm{~mm}$ to the lateral side (SD 0.9, range 0.95-4.19). The standardized ankle movement from $10^{\circ}$ plantar flexion and inversion to the $5^{\circ}$ dorsiflexion resulted in an mean nerve movement of $3.6 \mathrm{~mm}$ to the lateral side (SD 1.8, range 1.38-7.68). Both displacements to the lateral side were significant $(P<0.01)$ (Table 1).

The inter-observer reliability coefficients (ICC) for the measurement of nerve displacement for both ankle movements were 0.98 (95\% CI; 0.953-0.998) and 0.99 (95\% CI; $0.980-0.998)$, respectively.

With the ankle in the neutral position the superficial peroneal nerve or its terminal branches did not move while applying tension on the skin, flexion of the 4 th toe or flexion of all toes.

\section{Discussion}

The most important finding of the present study is that the anatomical orientation of the superficial peroneal nerve consistently changes by altering the ankle position. The superficial peroneal nerve, or if divided the intermediate dorsal cutaneous nerve, and the medial dorsal cutaneous nerve, are the only nerves in the human body that can be made visible in the majority of humans [21]. Numerous manoeuvres have been described to visualize the superficial peroneal nerve or its terminal branches $[3,7,9,13,17$, $21,24]$. These were introduced to attempt avoidance of iatrogenic damage during anterolateral portal placement, external fixator applications, surgical decompressions and rapid localization prior to local anaesthetic blocks [21]. One of these manoeuvres includes combined plantar flexion and inversion of the ankle [9, 17, 24].

The possibility to visualize the superficial peroneal nerve has clinical implications in ankle surgery (open and arthroscopically) and in local regional anaesthetic techniques. As described, different manoeuvres were introduced to visualize this nerve, being able to mark its course preoperatively, with the aim of preventing complications. Despite the possibility to visualize the nerve, complication rates in ankle arthroscopy are still relatively high, ranging from $8-17 \%$, mostly regarding the superficial peroneal nerve and especially the intermediate dorsal cutaneous nerve $[2,4,6,8-11,13,15,17,18,20]$.

One of the possible limitations of our research was that the specimens were amputated below the knee. The present investigation concerns the movement of the superficial peroneal nerve in different foot and ankle positions, in below the knee amputated specimens. We assumed that with the amputation the normal nerve anatomy might was disturbed, and for this reason the nerve was fixed to its surrounding soft tissue with a mosquito clamp. However, we hypothesize that the amputation did not have a major influence on our results, since from proximal to distal the nerve divides several times to innervate the peroneal muscles. Each division individually acts as an anchor, thereby stabilizing the nerve. The nerve is furthermore fixed along its route by the lateral intermuscular septum.

Marking the superficial peroneal nerve in ankle plantar flexion and inversion provides a false sense of safety when making the anterolateral portal in the normal or slight dorsiflexion position, since the marking does not correspond to the subcutaneous position of the nerve in this position. When the ankle is moved from plantar flexion and inversion to the neutral position $\left(90^{\circ}\right)$, the nerve consistently moves lateral relatively to the marking. It is this neutral or slightly dorsiflexed position in which the portals are created [26]. If the anterolateral portal is made medial to the marking, this will 
Table 1 General specimen characteristics with superficial peroneal nerve movement $(\mathrm{mm})$ from combined $10^{\circ}$ plantar flexion and inversion to neutral and to $5^{\circ}$ ankle dorsiflexion

\begin{tabular}{|c|c|c|c|c|c|c|c|c|c|c|}
\hline \multirow[t]{3}{*}{ Specimen } & \multirow[t]{3}{*}{ Age } & \multirow[t]{3}{*}{ Gender } & \multicolumn{8}{|c|}{ Superficial peroneal nerve movement $(\mathrm{mm})$ from $10^{\circ}$ plantar flexion and inversion to } \\
\hline & & & \multicolumn{4}{|l|}{ Neutral } & \multicolumn{4}{|c|}{$5^{\circ}$ dorsiflexion } \\
\hline & & & Observer 1 & Observer 2 & Observer 3 & Average & Observer 1 & Observer 2 & Observer 3 & Average \\
\hline 1 & 79 & 우 & 1.79 & 1.92 & 1.96 & 1.89 & 3.74 & 3.62 & 3.38 & 3.58 \\
\hline 2 & 83 & $\hat{o}$ & 1.64 & 1.57 & 1.68 & 1.63 & 1.89 & 2.09 & 1.81 & 1.93 \\
\hline 3 & 76 & 우 & 1.38 & 1.46 & 1.63 & 1.49 & 1.50 & 1.59 & 1.38 & 1.49 \\
\hline 4 & 71 & $\hat{o}$ & 0.95 & 1.00 & 0.99 & 0.98 & 1.95 & 1.91 & 1.75 & 1.87 \\
\hline 5 & 65 & $\hat{\sigma}$ & 3.22 & 3.58 & 3.19 & 3.33 & 3.25 & 3.45 & 3.86 & 3.52 \\
\hline 6 & 81 & $\hat{0}$ & 2.49 & 2.46 & 2.37 & 2.44 & 3.62 & 3.55 & 3.33 & 3.50 \\
\hline 7 & 90 & $\hat{\sigma}$ & 3.13 & 2.96 & 3.09 & 3.06 & 6.09 & 6.18 & 6.12 & 6.13 \\
\hline 8 & 72 & $\hat{\sigma}$ & 2.04 & 2.32 & 2.33 & 2.23 & 3.27 & 3.25 & 3.02 & 3.18 \\
\hline 9 & 67 & 우 & 2.60 & 2.54 & 2.40 & 2.51 & 3.33 & 3.29 & 3.49 & 3.37 \\
\hline 10 & 78 & $\hat{\sigma}$ & 3.98 & 3.92 & 4.19 & 4.03 & 7.49 & 7.39 & 7.68 & 7.52 \\
\hline \multirow[t]{4}{*}{ Median } & 77 & & & & Average & 2.36 & & & & 3.61 \\
\hline & & & & & SD & 0.92 & & & & 1.97 \\
\hline & & & \multicolumn{3}{|c|}{ ICC (Intraclass correlation coefficient) } & 0.98 & & & & 0.99 \\
\hline & & & \multicolumn{3}{|c|}{ One samplet test $(P<0.05)$} & $<0.01$ & & & & $<0.01$ \\
\hline
\end{tabular}

prevent iatrogenic damage to the superficial peroneal nerve. Placing the anterolateral portal lateral to the marking places the nerve at risk to iatrogenic damage.

\section{Conclusion}

The current study shows that the superficial peroneal nerve consistently moves lateral when the ankle is manoeuvred from combined plantar flexion and inversion to the neutral or dorsiflexed position. If visible, it is therefore advised to create the anterolateral portal medial from the preoperative marking, in order to prevent iatrogenic damage to the superficial peroneal nerve.

Open Access This article is distributed under the terms of the Creative Commons Attribution Noncommercial License which permits any noncommercial use, distribution, and reproduction in any medium, provided the original author(s) and source are credited.

\section{References}

1. Adkison DP, Bosse MJ, Gaccione DR et al (1991) Anatomical variations in the course of the superficial peroneal nerve. J Bone Joint Surg Am 73:112-114

2. Amendola A, Petrik J, Webster-Bogaert S (1996) Ankle arthroscopy: outcome in 79 consecutive patients. Arthroscopy 12:565-573

3. Barber FA, Britt BT, Ratliff HW et al (1988) Arthroscopic surgery of the ankle. Orthop Rev 17:446-451

4. Barber FA, Click J, Britt BT (1990) Complications of ankle arthroscopy. Foot Ankle 10:263-266
5. Blair JM, Botte MJ (1994) Surgical anatomy of the superficial 371 peroneal nerve in the ankle and foot. Clin Orthop Relat Res 305:229-238

6. Bonnin M, Bouysset M (1999) Arthroscopy of the ankle: analysis of results and indications on a series of 75 cases. Foot Ankle Int 20:744-751

7. Carson WG Jr, Andrews JR (1987) Arthroscopy of the ankle. Clin Sports Med 6:503-512

8. Cutsuries AM, Saltrick KR, Wagner J et al (1994) Arthroscopic arthroplasty of the ankle joint. Clin Podiatr Med Surg 11:449-467

9. Ferkel RD, Heath DD, Guhl JF (1996) Neurological complications of ankle arthroscopy. Arthroscopy 12:200-208

10. Ferkel RD, Small HN, Gittins JE (2001) Complications in foot and ankle arthroscopy. Clin Orthop Relat Res 391:89-104

11. Frey C, Feder KS, DiGiovanni C (1999) Arthroscopic evaluation of the subtalar joint: does sinus tarsi syndrome exist? Foot Ankle Int 20:185-191

12. Golano P, Vega J, Perez-Carro L, Gotzens V (2006) Ankle anatomy for the arthroscopist. Part I: the portals. Foot Ankle Clin $11: 253-273$

13. Harty M (1985) Ankle arthroscopy: anatomical features. Orthopedics 8:1538-1540

14. Horwitz MT (1938) Normal anatomy and variations of the peripheral nerves of the leg and foot. Application in operations for vascular diseases: study of one hundred specimens. Arch Surg $36: 626-636$

15. Jerosch J, Schneider T, Strauss JM et al (1993) Arthroscopy of the upper ankle joint. List of indications from the literaturerealistic expectations-complications. Unfallchirurg 96:82-87

16. Lamy C, Stienstra JJ (1994) Complications in ankle arthroscopy. Clin Podiatr Med Surg 11:523-539

17. Lerman BI, Gornish LA, Bellin HJ (1984) Injury of the superficial peroneal nerve. J Foot Surg 23:334-339

18. Martin DF, Baker CL, Curl WW et al (1989) Operative ankle arthroscopy. Long-term followup. Am J Sports Med 17:16-23

19. Saito A, Kikuchi S (1998) Anatomic relations between ankle arthroscopic portal sites and the superficial peroneal and saphenous nerves. Foot Ankle Int 19:748-752 
20. Scranton PE Jr, McDermott JE (1992) Anterior tibiotalar spurs: a comparison of open versus arthroscopic debridement. Foot Ankle 13:125-129

21. Stephens MM, Kelly PM (2000) Fourth toe flexion sign: a new clinical sign for identification of the superficial peroneal nerve. Foot Ankle Int 21:860-863

22. Stetson WB, Ferkel RD (1996) Ankle arthroscopy: I. Technique and complications. J Am Acad Orthop Surg 4:17-23

23. Takao M, Ochi M, Shu N et al (2001) A case of superficial peroneal nerve injury during ankle arthroscopy. Arthroscopy $17: 403-404$
24. Takao M, Uchio Y, Shu N et al (1998) Anatomic bases of ankle arthroscopy: study of superficial and deep peroneal nerves around anterolateral and anterocentral approach. Surg Radiol Anat 20:317-320

25. Unger F, Lajtai G, Ramadani F et al (2000) Arthroscopy of the upper ankle joint. A retrospective analysis of complications. Unfallchirurg 103:858-863

26. van Dijk CN, Scholte D (1997) Arthroscopy of the ankle joint. Arthroscopy 13:90-96

27. van Dijk CN, van Bergen CJ (2008) Advancements in ankle arthroscopy. J Am Acad Orthop Surg 16:635-646 